\title{
DRIVER BEHAVIOR AS A FUNCTION OF AMBIENT LIGHT AND ROAD GEOMETRY
}

\author{
Michael J. Flannagan \\ John M. Sullivan \\ Scott E. Bogard \\ University of Michigan Transportation Research Institute \\ 2901 Baxter Road \\ Ann Arbor, MI 48109 USA \\ E-mail: mjf@umich.edu
}

\begin{abstract}
\section{OBJECTIVES}

To determine how ambient light (day versus night) and road geometry affect driving behavior, especially the speeds that drivers choose when not constrained by lead vehicles.
\end{abstract}

\section{METHODS}

Recently, it has become technically easier to observe how people drive $b$ offering them longterm use of highly instrumented vehicles. Much of this type of work has been done in connection with large-scale field operational tests (FOTs) of various innovative vehicle systems. The information obtained is in many ways complementary to information from observation of traffic. Traffic observation often provides information about a large number of drivers, but at a relatively coarse level and in a spatially and temporally limited context (i.e., observing how a large number of drivers negotiate a particular intersection). In contrast, long-term use of highly instrumented vehicles is more restricted in terms of how many drivers can be observed, although the feasible numbers are now reasonably high. On the positive side, data from instrumented vehicles can offer very detailed information about driving behavior over many miles and many days.

In this paper, we present results from a database of driving behavior that was derived from a recent FOT for an adaptive cruise control (ACC) system (although the data used here are all from phases of the study that involved only normal vehicle equipment). The FOT involved ten identical cars that were instrumented for a variety of types of data. The most important data for present purposes were: speed, yaw rate, location from the Global Positioning System (GPS), and presence or absence of a lead vehicle within about $100 \mathrm{~m}$ based on the forward-looking sensors of the ACC system. The instrumented cars were driven by a total of 108 participants, each of whom was given a car to use as his or her own vehicle in normal driving for either two or five weeks. The participants were sampled from licensed drivers in southeastern Michigan, and represented a wide range of age and driving experience.

\section{RESULTS}

Results will be reported in terms of speed as a function of horizontal road curvature in light and dark conditions, and as a function of driver age and gender, all for situations in which there is no lead vehicle within about $100 \mathrm{~m}$. 


\section{CONCLUSIONS}

Current evidence about headlighting suggests that drivers' ability to see and negotiate the roadway is virtually unaffected by differences in ambient light, although their ability to perceive and avoid objects on the road, such as pedestrians, is greatly reduced when headlamps are the main source of light. There is also evidence that drivers do not markedly reduce their speed in conditions of low ambient light. The current analysis allows us to determine how drivers react to specific road geometries in light and dark conditions. This has implications for how well drivers' perceptual abilities match their driving behavior, and also for assessing the potential benefit of a variety of innovative headlighting systems that are currently being designed to adapt in various ways to vehicle speed and road geometry. 